\title{
Application of Beamforming in Wireless Location Estimation
}

\author{
Kamran Sayrafian-Pour ${ }^{1}$ and Dominik Kaspar ${ }^{2}$ \\ ${ }^{1}$ National Institute of Standard and Technology, Gaithersburg, MD 20899, USA \\ ${ }^{2}$ Department of Computer Science, Swiss Federal Institute of Technology, Zurich, Switzerland
}

Received 1 June 2005; Revised 27 November 2005; Accepted 1 December 2005

\begin{abstract}
A simple technique to estimate the position of a given mobile source inside a building is based on the received signal strength. For this methodology to have a reasonable accuracy, radio visibility of the mobile by at least three access points is required. To reduce the number of the required access points and therefore simplify the underlying coverage design problem, we propose a novel scheme that takes into account the distribution of RF energy around the receiver. In other words, we assume that the receiver is equipped with a circular array antenna with beamforming capability. In this way, the spatial spectrum of the received power can be measured by electronically rotating the main lobe around the 360-degree field of view. This spatial spectrum can be used by a single receiver as a means for estimating the position of the mobile transmitter. In this paper, we investigate the feasibility of this methodology, and show the improvement achieved in the positioning accuracy.
\end{abstract}

Copyright (C) 2006 K. Sayrafian-Pour and D. Kaspar. This is an open access article distributed under the Creative Commons Attribution License, which permits unrestricted use, distribution, and reproduction in any medium, provided the original work is properly cited.

\section{INTRODUCTION}

In recent years, technologies that find the location of mobile sources inside buildings are becoming an attractive area of research and development. A significant application of such technologies is in emergency situations where it is important to be able to locate or track the movements of the first responders inside closed environments. More commercial and public safety applications are also emerging every day.

GPS provides this capability in the outdoor environment, where the line-of-sight propagation paths to GPS satellites exist. However, it cannot be used in the indoor environment where ceilings obstruct the view of the corresponding satellites. The problem of finding locations of mobile sources inside buildings presents special challenges. Obstacles such as walls, furniture, and other objects create a much harsher radio propagation environment. A variety of ranging and positioning techniques with different technologies such as RF, ultrasound, infrared, DC electromagnetic, and so forth, have been proposed to solve this problem [1]. Accordingly, various levels of localization accuracy, resolution, and complexity have been reported by such methodologies.

A simple technique to estimate the position of a given source is based on the received signal strength (RSS). RSS is attractive because it is widely applicable to wireless sensor networks and does not require sophisticated localization hardware. The general philosophy in this approach is to establish a one-to-one correspondence between a given position and the average received signal strength from at least three transmitters with known locations. One such system that has been implemented on the existing wireless local area network infrastructure is RADAR [2].

RADAR is a software-based localization system that operates by recording and processing RSS information from multiple access points (i.e., base stations). There are two main phases in the operation of this system: an off-line phase (i.e., data collection or training phase) and an online phase (i.e., mobile position estimation). In the off-line phase, a "radio-map" of the environment is created. A "radio-map" is a database of selected locations and their corresponding received signal strengths from several base stations. For example, an entry in the radio-map may look like $(x, y, z$, $\left.\operatorname{RSS}_{i(i=1,2, \ldots, n)}\right)$, where $(x, y, z)$ is the physical coordinates of the location where the signal is recorded and $\mathrm{RSS}_{i}$ is the average received signal strength of the base station " $i$." In the on-line phase, the mobile measures the received signal strength from each of the base stations within range, and then, searches through the radio-map database to determine the best signal strength vector that matches the one observed. The system estimates the location associated with the best-matching signal strength vector (i.e., nearest neighbor) to be the location of the mobile. This technique essentially calculates the $L_{2}$ distance (i.e., euclidean distance) between the observed RSSs and the entries in the set defined by the 


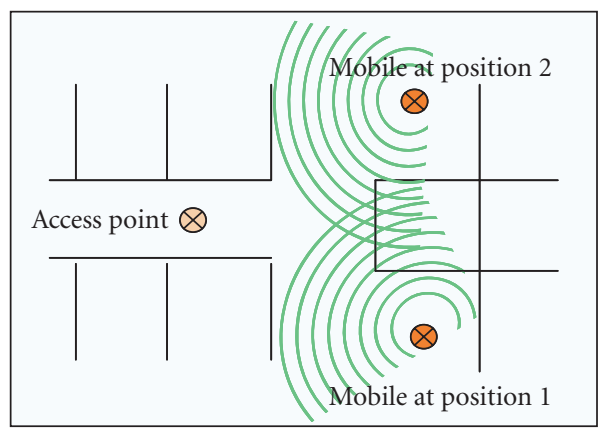

FIGURE 1: Ambiguity in mobile position with one access point using RSS.

radio-map. It then picks the RSS-vector that minimizes this distance and declares the corresponding physical coordinate as the estimate of the mobile's location. Alternative strategies such as averaging the k-nearest neighbors have also been considered.

Another interesting RSS-based localization methodology has been proposed in $[3,4]$, where a probability distribution is constructed during the training phase. Then, a Bayesian inference approach is used to estimate the mobile's coordinates with the highest probability.

In [5], fundamental limits of localization using RSS in indoor environments have been characterized. It is shown that using commodity 802.11 technology over a range of algorithms, approaches, and environments, one can expect a median localization error of $3 \mathrm{~m}$ and $97 \mathrm{th}$ percentile of $9 \mathrm{~m}$. It is also argued that these limitations are fundamental and that they are unlikely to transcend without a fundamentally more complex environmental models, additional localization infrastructure, or resources.

The general assumption in all of the RSS-based positioning systems is that the signal strength is recorded with an omnidirectional antenna at the receiver. In a multipath environment, such as indoor, the mobile receives the transmitted signal from many directions due to possible reflections, diffractions and scattering phenomena. An omnidirectional antenna is not capable of obtaining any information regarding the spatial (i.e., angular) distribution of the signal energy. The thesis of this research is that any information pertaining to the angular distribution of power can be used to increase the accuracy of an RSS-based localization methodology. For example, through the use of an antenna that has beamforming capability, more information can be extracted by measuring the signal strength in different directions; therefore, instead of the average signal power, a more general and sophisticated spatial power spectrum (SPS) can be generated and used for position estimation.

For example, in Figure 1, due to symmetry, the access point experiences the same average received signal power from a mobile located at position 1 or 2; therefore, with a single access point, no RSS-based positioning system will be capable of resolving the ambiguity between these positions. However, as observed, the directions from which the access

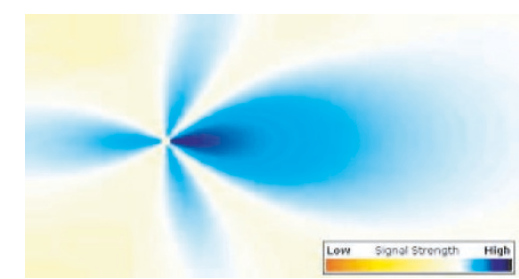

(a)

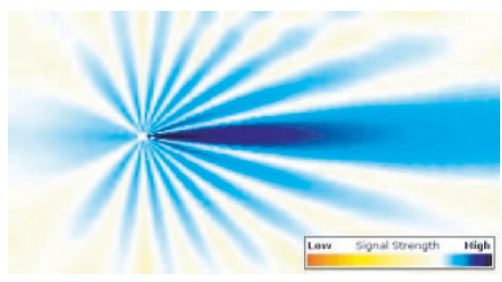

(b)

FIgURE 2: Beam pattern of a circular array with (a) 8 elements, (b) 32 elements.

point receives most of the transmitted power from these positions are very different. If the positioning system has access to this kind of information (i.e., received power expected from different directions), distinction between positions 1 and 2 can be easily made.

Consequently, by using a more generalized and sophisticated radio-map that contains received signal strength information from various directions, the system would have the capability of estimating the mobile position with fewer access points and possibly higher accuracy.

Section 2 will describe the problem statement in more details. Simulation platform and various proposed solutions are investigated in Section 3. System performance is discussed in Section 4 and finally some concluding remarks are expressed in Section 5.

\section{PROBLEM STATEMENT AND MODELING}

An array antenna with beamforming capability is able to steer the direction of its main beam toward any desired angle. In particular, a circular array, which has a 360-degree field of view, is an appropriate candidate for two-dimensional positioning application. Sample beam pattern of such an antenna for various array sizes (i.e., number of elements) is shown in Figure 2.

Here, we propose to follow the same two-phase approach as the general RSS-based localization mentioned in the previous section. However, in the training phase, instead of recording the received signal strength, a circular array antenna with beamforming capability records the spatial power spectrum (SPS) of the received signal. The SPS is basically a two-dimensional graph of the received power versus angle (e.g., azimuth). Each point on this graph indicates the received signal strength when the main beam of the antenna is directed toward the corresponding azimuth. The beam of the array antenna is electronically controlled to point toward a 


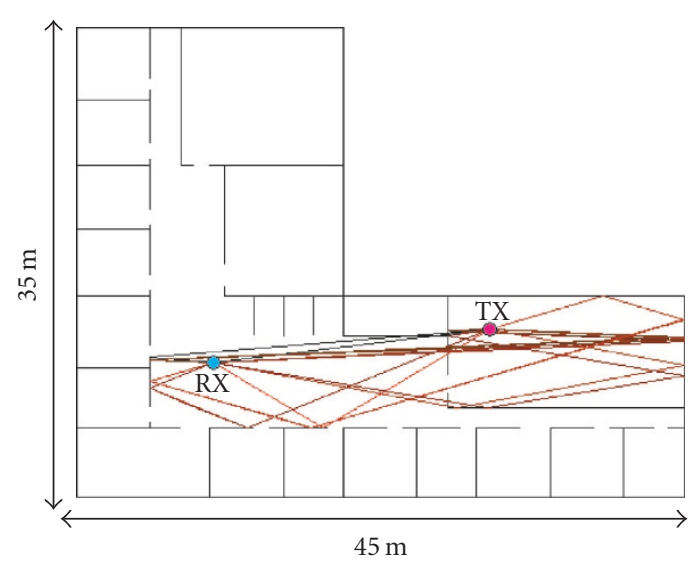

- Transmitter

- Receiver

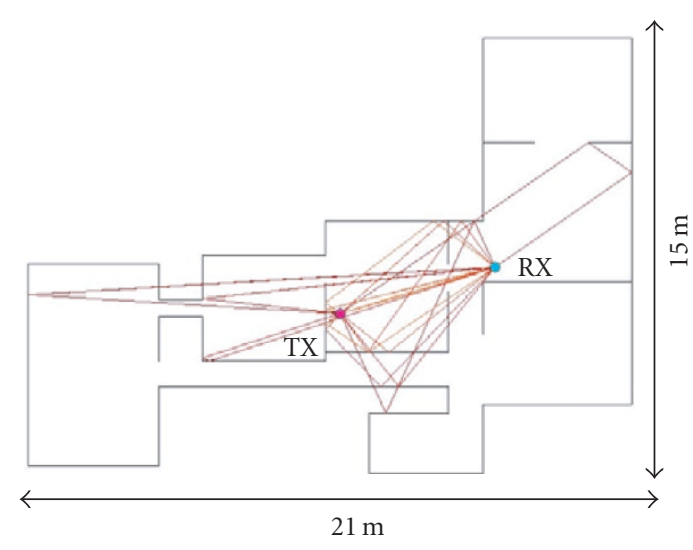

- Transmitter

- Receiver

(a)

(b)

FIgURE 3: Sample output of the ray-tracing tool for (a) building 1, (b) building 2.

desired direction. Therefore, by rotating the main lobe in the 360 -degree field of view and recording the received power, an SPS graph for a given mobile position can be generated.

Now, the problem is to first form a database of the measured spectra at the points of interest (e.g., set of grid points over the layout). This is the training phase which essentially yields a more sophisticated radio-map of the building where positioning is desired. Next, for any given position, the generated SPS can be compared to all the entries in this database, and the position of the best match would be a good candidate for the unknown position.

In this paper, we investigate the feasibility of this approach by implementing a simulation platform that matches the condition of an indoor environment. The main difficulty in simulating an indoor RF channel is the strong dependence of the received signal on the layout of the building (e.g., multipath channel). In particular, all walls, windows, and other objects that affect the propagation of RF waves will directly impact the signal strength and more importantly the directions from which the RF signal is received. Empirical, statisti$\mathrm{cal}$, and deterministic models have been used to describe the behavior of such multipath channels [6-8]. In our study, we have elected to use a sophisticated ray-tracing tool to accurately predict the received signal in the indoor RF channel. Wireless system engineering (WiSE) is a ray-tracing tool that has been developed and verified by Bell Laboratories $[9,10]$.

Figure 3 shows a pictorial sample of the multipath signal for a given building layout and transmitter-receiver location obtained through the ray-tracing tool. We realize that even such models have limitations in their accuracy and are also subject to errors when there are changes in the environment such as furniture moving, or even people walking through the building; however, this approach will give us the opportunity to create a testbed that to the extent possible mimics the conditions of an indoor channel in real life.
The received power in an array antenna with a directional beam is a function of the azimuth angle where the main beam is pointing. For a given layout, building material, transmitter-receiver location, frequency, and array size, the spatial power spectrum (SPS) at the receiver coordinates can be obtained by rotating the main beam around the receiver using a beamforming algorithm. In order to further verify the accuracy of the obtained SPS, we also conducted a simple experiment to compare sample hardware measurements to the predicted values of the ray-tracing tool (see the appendix for more details).

Once the SPS data for a set of predetermined points is collected, the test and verification phase of the positioning system can begin. Essentially each SPS graph can be regarded as a spatial signature that signifies the position coordinates of the mobile as seen by an access point. On the contrary to the RSS-based methodology, where $L_{2}$ distance is used to establish a metric between two RSS vectors, the problem of finding the closest match to a given SPS is not so evident. To further elaborate on this problem, consider the scenarios depicted in Figures 4(a) and 5(a). Here, the mobile is the transmitter (with a simple omnidirectional antenna) and the access point is the receiver equipped with a circular array antenna. Figure 4(b) displays the SPS observed by the access point when the mobile position changes from 0 to 1 . Since, the mobile distance from the access point is increased, the SPS graph decreases in magnitude (i.e., vertical shift) while generally maintaining its shape.

Figure 5(a) shows the scenario where the mobile changes its position from 0 to 2 . In this case, the distance of the mobile to the access point is almost unchanged; however, the direction from which the access points receive the RF signal is now changed. This translates to a horizontal shift in the spatial signature as seen in Figure 5(b). 


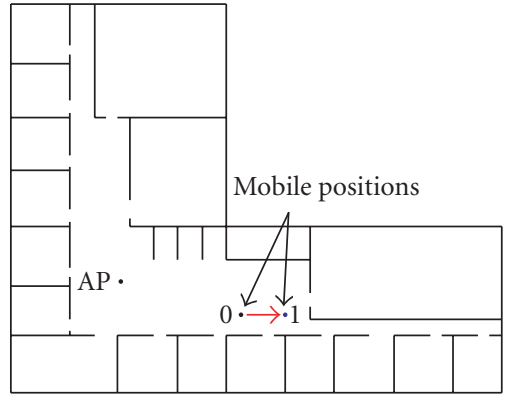

(a)

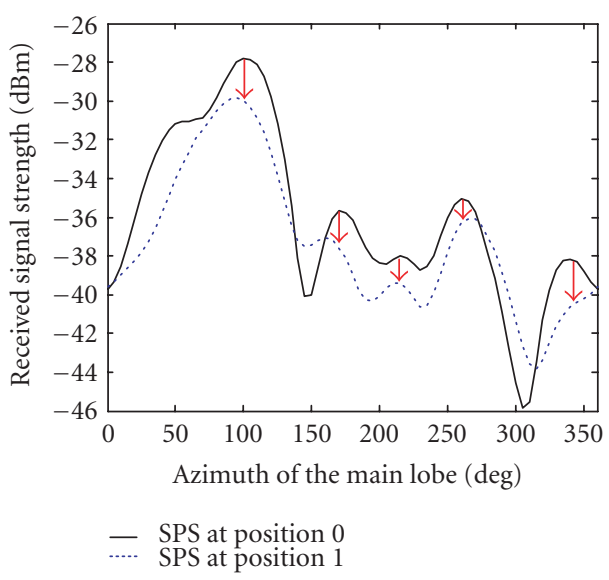

(b)

FIGURE 4: Variation of the spatial signature.

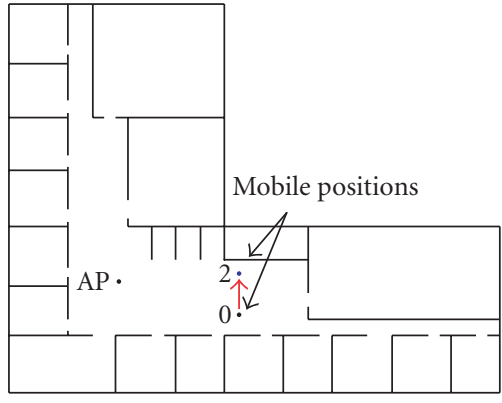

(a)

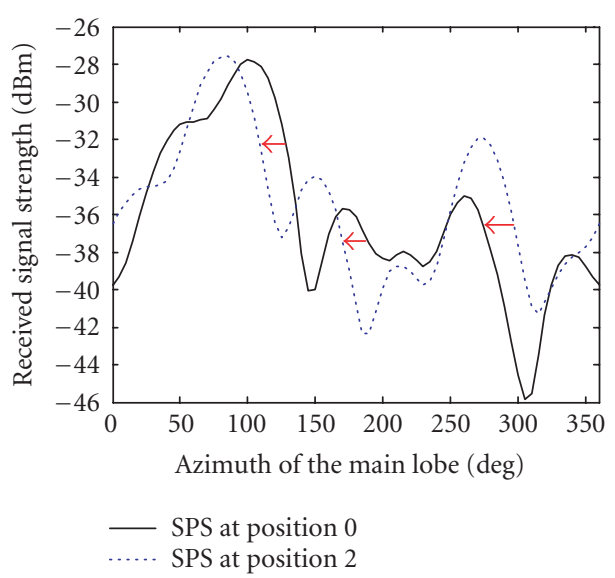

(b)

FIGURE 5: Variation of the spatial signature.
Therefore, physical closeness or proximity in mobile positions could translate to visual similarity in the spatial signatures seen by an access point. Although, it can be shown that this is not true in all cases, the methodology outlined in this paper is still applicable under all circumstances.

In order to measure the similarity between two signatures (i.e., matching), a distance metric has to be chosen that is capable of considering both of the situations above. A few metrics with this capability will be described in Section 3.

\section{SIMULATION PLATFORM}

The block diagram shown in Figure 6 describes the simulation system that was created to assess the performance of this positioning technique. Performance can be obtained for various input parameters such as building layouts, radio characteristics of the building materials (e.g., dielectric properties of the walls), and receiver-transmitter attributes such as power, frequency, and antenna gain pattern. Also, various signature-matching strategies can be implemented as search mechanisms to identify the position estimate of the mobile.

To generate a radio-map for a given layout, we have defined a grid of points as seen in Figure 7. Points that are too close to the walls are eliminated to preserve the possibility of future practical implementation. For each point on the grid and for each access point, a spatial signature is generated and stored. This constitutes the radio-map. Notice that if the antenna gain pattern for the receiver is taken to be omnidirectional, then the system will behave similar to the RSS-based positioning (e.g., [2]). This special case is actually used as a benchmark to evaluate the gain associated with using spatial spectra.

As previously mentioned, the main objective in this research is to study the applicability of using spatial power spectrum for indoor localization. In order to compare the signature of a test point to those included in the radio-map, an appropriate distance metric needs to be defined. We have considered various metrics that are briefly described in the following subsections. Performance of the system with each metric will then be compared to the omnidirectional case under various scenarios and parameters such as transmitter and receiver locations, building layout, number of receivers (i.e., access points), and so forth.

\subsection{Minkowski distance}

Minkowski metrics are a family of distance measures, which are generalized from the euclidean distance formula. It is often used as a similarity measure between two patterns that could be images, graphs, signatures, or vectors. If $d_{L_{r}}\left(\mathrm{SPS}_{1}\right.$, 


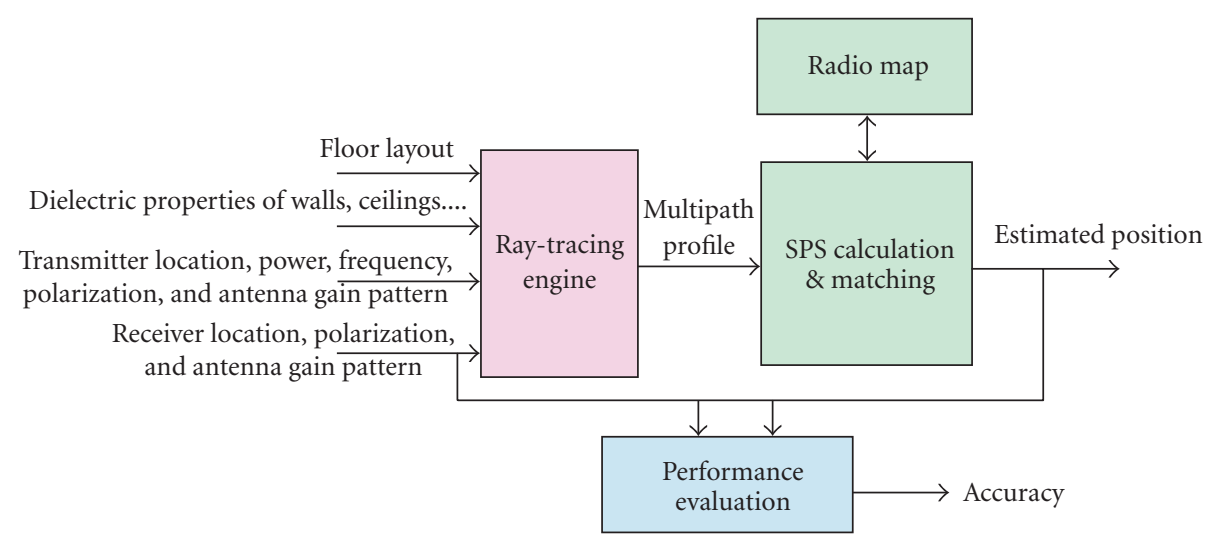

Figure 6: Block diagram of the simulation platform.
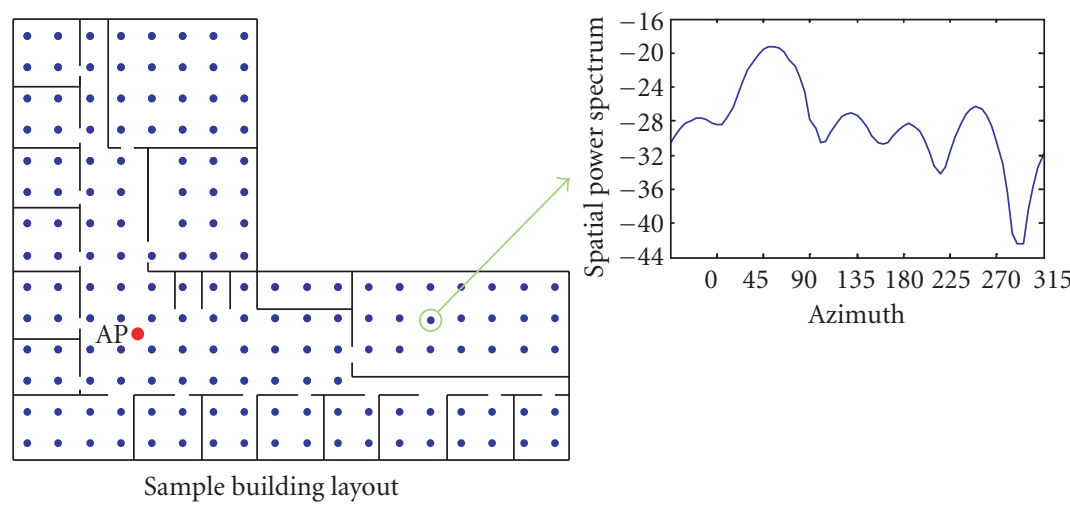

FIgURE 7: Radio-map generation based on a grid overlay.

$\mathrm{SPS}_{2}$ ) denotes the distance between two signatures $\mathrm{SPS}_{1}$ and $\mathrm{SPS}_{2}$, then Minkowski distance of order " $r$ " is defined as

$$
d_{L_{r}}\left(\mathrm{SPS}_{1}, \mathrm{SPS}_{2}\right)=\left(\sum_{\theta}\left|\operatorname{SPS}_{1}(\theta)-\mathrm{SPS}_{2}(\theta)\right|^{r}\right)^{1 / r}
$$

At $r=2$, this metric is the typical Euclidean distance that has been used for some of the RSS-based methodologies [2]. We chose to investigate the performance of $L_{1}$ and $L_{2}$ distance metrics for the spatial spectrum matching. These metrics essentially perform an element-by-element similarity measure between the two signatures $\mathrm{SPS}_{1}$ and $\mathrm{SPS}_{2}$, which might be less accurate for signatures that are circularly shifted versions of each other. This could be especially important when the radio-map grid resolution is low or there exist large open spaces in the layout (e.g., large conference rooms). An example will be provided later in Section 4 to further elaborate on this point.

\subsection{Earth mover algorithm (EMD)}

Earth mover's distance (EMD) has been used as a distance metric with application in content-based image retrieval [11]. An attractive property of this metric is its capability to match perceptual similarity better than other distance metrics used for image retrieval. This property is actually desirable in our application as well, since in most cases perceptual matching of spatial signatures (i.e., SPS) would seem to apply in actual coordinate matching for indoor positioning.

The EMD is based on a solution to the transportation problem from linear optimization. It is a useful and flexible distance metric that measures the minimal cost that must be paid to transform one signature into the other. Signature matching is cast as a transportation problem by defining one signature as the supplier and the other as the consumer, and by setting the cost for a supplier-consumer pair to equal the ground distance between an element in the first signature and an element in the second. Intuitively, the solution is the minimum amount of work required to transform one signature into the other. Alternatively, given two spatial spectra, one can be seen as a mass of earth properly spread in space, the other as a collection of holes in that same space. Then, the EMD measures the least amount of work needed to fill the holes with earth. A unit of work corresponds to transporting a unit of earth by a unit of ground distance.

We have investigated the performance of this metric as a similarity measure between two spatial spectra. 


\subsection{Hausdorff distance (HD)}

Hausdorff Distance is a measure of closeness of two sets of geometric points $P$ and $Q[12,13]$ and is defined as

$$
\begin{aligned}
& \operatorname{HD}(P, Q) \\
& \quad=\max \left(\max _{a \in P} \min _{b \in Q}(\|a-b\|), \max _{a \in Q} \min _{b \in P}(\|a-b\|)\right) .
\end{aligned}
$$

In this case, we would like to measure the distance between the two functions $\operatorname{SPS}_{1}(\theta), \operatorname{SPS}_{2}(\theta)$. First, we define the points $a_{\theta}$ and $b_{\theta}$ with the following coordinates:

$$
a(\theta)=\left(\theta, \operatorname{SPS}_{1}(\theta)\right), \quad b(\theta)=\left(\theta, \operatorname{SPS}_{2}(\theta)\right) .
$$

Then, we customize the definition of Hausdorff distance as follows:

$$
\begin{array}{r}
\mathrm{HD}\left(\mathrm{SPS}_{1}, \mathrm{SPS}_{2}\right)=\max \left(\max _{\theta_{1}} \min _{\theta_{2}}\left(\left\|a\left(\theta_{1}\right)-b\left(\theta_{2}\right)\right\|\right),\right. \\
\left.\max _{\theta_{2}} \min _{\theta_{1}}\left(\left\|a\left(\theta_{1}\right)-b\left(\theta_{2}\right)\right\|\right)\right),
\end{array}
$$

where

$$
\begin{aligned}
& \left\|a\left(\theta_{1}\right)-b\left(\theta_{2}\right)\right\| \\
& =d_{L_{2}}\left(a\left(\theta_{1}\right), b(\theta)\right) \\
& =\left(\left(\theta_{1}-\theta_{2}\right)^{2} c+\left(\operatorname{SPS}_{1}\left(\theta_{1}\right)-\operatorname{SPS}_{2}\left(\theta_{2}\right)\right)^{2}\right)^{1 / 2}
\end{aligned}
$$

and " $c$ " is a constant scaling factor chosen appropriately.

Hausdorff distance measures the degree of mismatch between two sets, as it reflects the distance of the points in the first set that is furthest from any point in the second set. Intuitively, if the Hausdorff distance is $d$, then every point of the first set must be within a distance $d$ of some point of the second set and vice versa. Hausdorff distance obeys the properties of identity, symmetry, and triangle inequality; therefore, it is a metric over the set of all closed and bounded sets. Hausdorff distance has been used as a metric to develop fast and reliable method for comparing binary images and locating objects within images $[14,15]$. Here, we would like to investigate its applicability to establish a similarity measure between two spatial signatures.

\subsection{Kullback-Leibler distance (KL)}

The Kullback-Leibler distance (or relative entropy) is a natural distance function from a "true" probability distribution $p$ to a "target" probability distribution $q$. For discrete probability distributions, $p=\left\{p_{1}, p_{2}, \ldots, p_{n}\right\}$ and $q=q_{1}, q_{2}, \ldots, q_{n}$, the KL-distance is defined to be [16]

$$
\mathrm{KL}(p, q)=\sum_{i} \log _{2}\left(\frac{p_{i}}{q_{i}}\right) .
$$

KL distance has been used as an objective measure that is able to predict audible discontinuities in concatenative speech

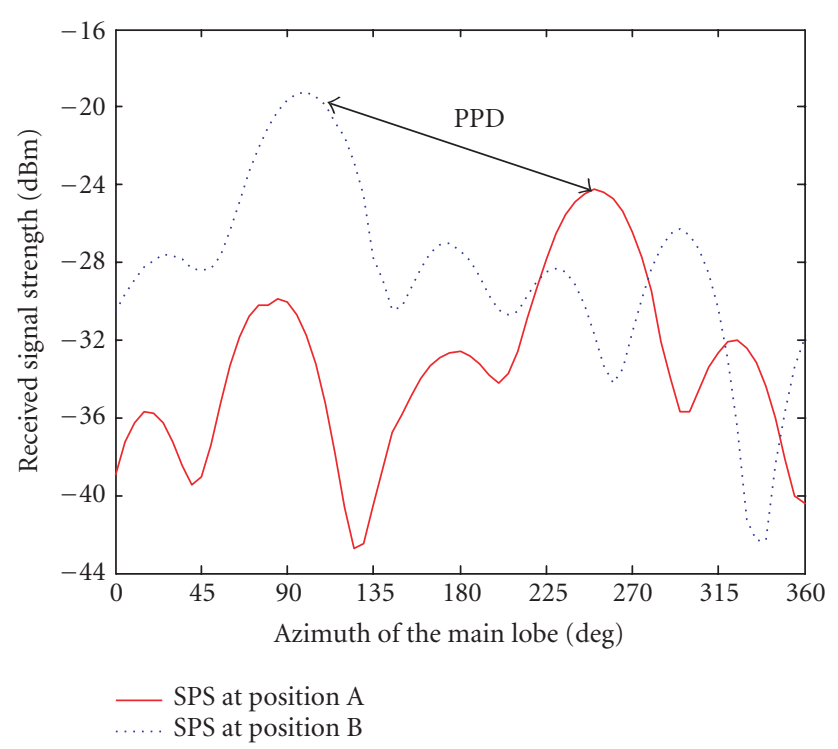

FIGURE 8: Example of a peak-to-peak distance.

synthesis [17]. It has also been used as a similarity measure between images [18]. Here, we would like to investigate its effectiveness as a similarity measure between two spatial spectra. Therefore, we use the following expression as a metric that quantifies the distance between two spatial spectra:

$$
\mathrm{KL}\left(\mathrm{SPS}_{1}^{*}, \mathrm{SPS}_{2}^{*}\right)=\sum_{\theta} \operatorname{SPS}_{1}^{*}(\theta) \log _{2}\left(\frac{\mathrm{SPS}_{1}^{*}(\theta)}{\operatorname{SPS}_{2}^{*}(\theta)}\right)
$$

Note that the KL-distance is not symmetric and SPS* is the normalized SPS.

\subsection{Peak-to-peak distance (PPD)}

The direction from which a node receives most of the transmitted RF energy is a function of the building layout and the position of the node. This direction is basically the azimuth angle where SPS peaks. Although, this peak might not be indicative of the transmitter's direction, it may be used to establish a distance metric between two spatial spectra; and therefore, help to estimate the coordinates of the mobile by finding the best match. Assume that $\theta_{1}$ and $\theta_{2}$ are the azimuth directions where $\mathrm{SPS}_{1}$ and $\mathrm{SPS}_{2}$ peak. In other words,

$$
\theta_{1}=\arg \max _{\theta} \operatorname{SPS}_{1}(\theta), \quad \theta_{2}=\arg \max _{\theta} \operatorname{SPS}_{2}(\theta) .
$$

Then, define the peak-to-peak distance (PPD) as

$$
\operatorname{PPD}=\left(\left(\theta_{1}-\theta_{2}\right)^{2} c+\left(\operatorname{SPS}_{1}\left(\theta_{1}\right)-\operatorname{SPS}_{2}\left(\theta_{2}\right)\right)^{2}\right)^{1 / 2},
$$

where " $c$ " is a constant scaling factor chosen appropriately.

Figure 8 displays an example of this distance. In terms of the complexity, this is a simple measure to implement. Since, now (instead of the whole SPS) only the values associated to each SPS peak can be precomputed and stored in the radiomap. 
TABLE 1: Average position error (in meters) for the layout of Figure 3(a) (array size $=8$, step-size $=5$ degrees).

\begin{tabular}{|c|c|c|c|c|c|c|c|c|c|}
\hline & \multicolumn{3}{|c|}{ Radio map res $1 \times 1$} & \multicolumn{3}{|c|}{ Radio map res $2 \times 2$} & \multicolumn{3}{|c|}{ Radio map res $3 \times 3$} \\
\hline & $\mathrm{AP}=1$ & $\mathrm{AP}=2$ & $\mathrm{AP}=3$ & $\mathrm{AP}=1$ & $\mathrm{AP}=2$ & $\mathrm{AP}=3$ & $\mathrm{AP}=1$ & $\mathrm{AP}=2$ & $\mathrm{AP}=3$ \\
\hline SPS-L1 & 0.83 & 0.82 & 0.82 & 3.1 & 2.12 & 2.12 & 5.05 & 4.11 & 2.77 \\
\hline SPS-L2 & 1.03 & 0.86 & 1.06 & 3.2 & 2.46 & 2.34 & 5.07 & 4.58 & 2.9 \\
\hline SPS-EMD & 1.05 & 1.04 & 1.02 & 3.22 & 2.28 & 2.25 & 5.95 & 4.49 & 3.07 \\
\hline SPS-PPD & 1.90 & 1.50 & 1.45 & 4.97 & 4.10 & 3.31 & 6.88 & 6.03 & 4.41 \\
\hline SPS-HD & 2.30 & 1.59 & 1.55 & 5.12 & 3.11 & 3.08 & 7.94 & 4.52 & 3.68 \\
\hline SPS-KL & 2.64 & 1.35 & 1.34 & 5.88 & 4.28 & 3.13 & 7.56 & 5.52 & 4.25 \\
\hline RSS-L2 & 15.78 & 4.37 & 2.5 & 16.04 & 5.54 & 4.32 & 16.37 & 6.66 & 5.53 \\
\hline
\end{tabular}

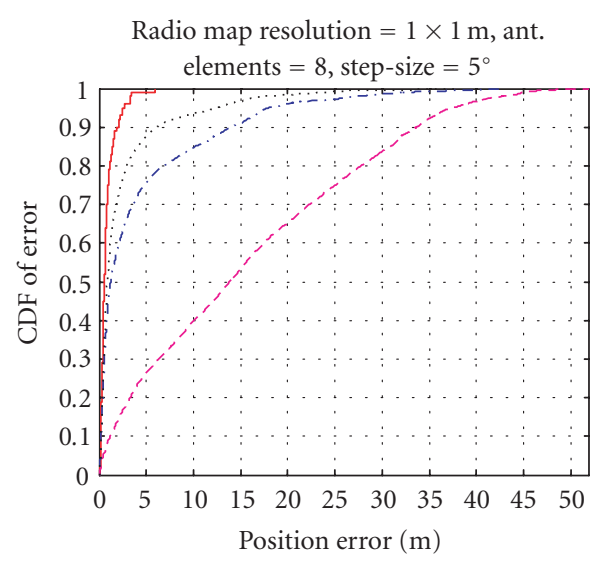

- SPS-based ( 1 access point)
- - - RSS-based ( 1 access point)
-.- RSS-based ( 2 access points)
… RSS-based ( 3 access points)

(a) Radio map resolution $1 \times 1 \mathrm{~m}$

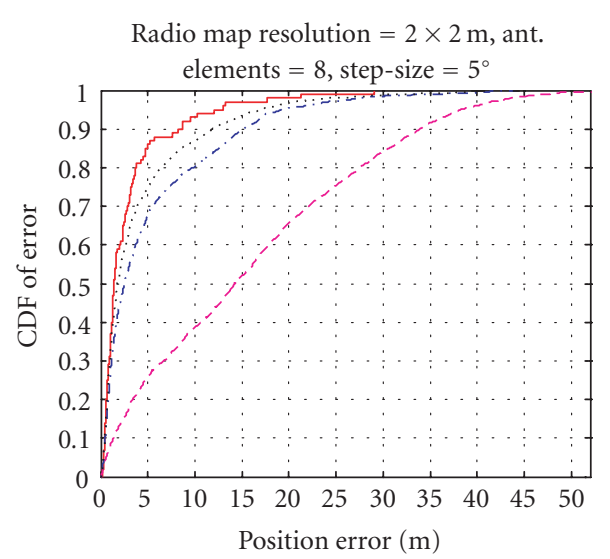

— SPS-based ( 1 access point)

- - - RSS-based ( 1 access point)

-..- RSS-based (2 access points)

RSS-based ( 3 access points)

(b) Radio map resolution $2 \times 2 \mathrm{~m}$

FIGURE 9: CDF of the position error (ant. elements $=8$, step-size $=5$ degrees).

\section{SYSTEM PERFORMANCE}

Using the simulation platform discussed in the previous section, we conducted experiments for various layouts, array sizes (i.e., number of elements), radio-map grid resolutions, antenna beam rotation step size and signature matching techniques. We have chosen an ISM-band frequency of $2.4 \mathrm{GHz}$ for the operation of the system in simulation.

Table 1 summarizes the average position error obtained with different matching algorithms, number of access points, and radio-map grid resolution for the layout shown in Figure 3(a). When the receiver antenna is selected to be omnidirectional (i.e., array size is one), we will essentially have an RSS-based system where no directional information is included in the signatures and the radio-map. In this case, each spatial spectrum is replaced by a scalar that represents the total average received power. Performance of this omnidirectional system with $L_{2}$ distance metric has been chosen as the benchmark for comparison purposes and it is displayed in the last row of Table 1. As observed, all SPS-based approaches significantly outperform the RSS method; with SPS-L1 having the least average error for this layout.
Figure 9 also displays the cumulative distribution function (CDF) of error in the estimated position for different radio-map resolution. For example, in Figure 9(a), the performance of the SPS-L1 method with only one access point has been compared to the performance of the RSS-based method with one, two, and three access points. As observed, using the SPS approach, there is a significant improvement in accuracy when the number of access points is less than three. Even in the case of RSS-based approach with 3 APs, the gain associated with SPS-L1 is noticeable when radio-map resolution is high (i.e., $1 \times 1 \mathrm{~m}$ ).

Figure 10 visually demonstrates the advantage of using spatial spectrum for lower number of access points.

For the above results, we have selected the position of the best matching point in the radio-map as the estimated position of the mobile. Selecting the k-nearest neighbors and averaging the results did not amount to significant difference; therefore, we did not reflect those results.

The average errors reported in Table 1 have been obtained by considering 400 test points uniformly distributed throughout the layout. The signal-to-noise-ratio (SNR) for these points varies from 15 to $75 \mathrm{~dB}$. Figure 11 demonstrates 


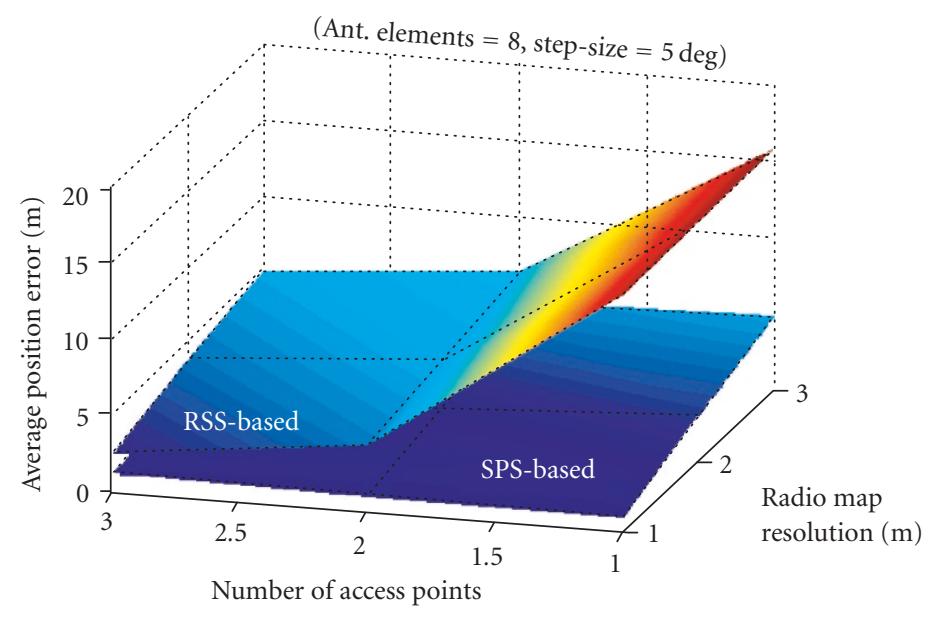

FIGURE 10: Advantage of using SPS.

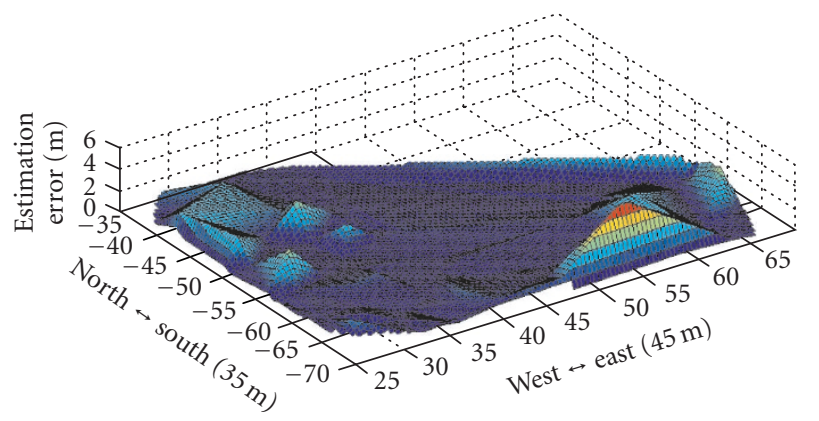

FIgURE 11: Absolute error of the test points over the layout of Figure 3(a).

the absolute error of each test point on a three dimensional map over the layout. As observed, test points that are located near the top left and bottom right corners of the building experience higher position error. It is important to note that mobiles located in these corners experience a lower SNR; this in turn contributes to higher error in their estimated position.

Another experiment was performed for the building layout shown in Figure 3(b) that has a physical dimension of $15 \times 21 \mathrm{~m}$ and a completely different wall material (i.e., sheetrock). The results are summarized in Table 2. A similar trend in terms of SPS advantage is also observed for this layout.

In case where the radio-map grid resolution is low or where there exist large open spaces in the layout (e.g., large conference rooms), the performance of SPS-based approach with Minkowski distance metric might be inferior to other matching techniques such as PPD or EMD. To explain this issue, consider a single large room with the size $16 \times 16 \mathrm{~m}$ and a single AP that is located in the middle of the room. Once again, we would like to estimate the position of a mobile transmitter that is moving around this room. Table 3 shows the average position error obtained by using various
SPS matching techniques. In this case, both EMD and PPD (i.e., highlighted rows in Table 3) outperform L1 and L2.

The last row of Table 3 indicates the average distance between the sample mobile position and the closest neighboring grid point that is located on the radio-map. This is the lowest possible error that can be achieved by any algorithm that only considers the best matching signature. It is interesting to note that the performance of PPD and EMD (i.e., highlighted rows) are very close to this lower bound.

A similar experiment was performed for even a larger room (i.e., $32 \times 32 \mathrm{~m}$ ) and coarser radio-map resolutions. The result (see Table 4) also pointed out to the same conclusion; both PPD and EMD provided higher accuracy compared to L1 and L2.

In general, we have observed that matching techniques such as PPD or EMD outperform Minkowski distance metrics in environments where angular spread of energy around the receiver is highly nonuniform. In such environments, these algorithms are more sensitive to horizontal shifts in SPS (as explained in Figure 5(b)); and therefore, generate better accuracy in response to a change in mobile position. On the other hand, metrics such as $L_{1}$ or $L_{2}$ exhibit better performance when there are no significant directions from which the RF energy is received.

The number of antenna array elements used for the simulation results in Tables $(1,2,3,4)$ is eight. As the number of array elements increases, the main lobe of the beam pattern becomes narrower as seen in Figure 2. The antenna in this case would be capable of measuring the fine-grained spatial multipath profile of the signal at the receiver location. However, it is not clear whether such fine-grained SPS would enhance the achieved positioning accuracy. For this reason, we have performed further studies to understand the effect of the antenna size on the average positioning error. We have observed that for a given radio-map resolution, building layout, and matching algorithm; there might exist an optimal array size that results in the minimum average position error. For the building in Figure 3(a) with a radio-map grid resolution of $3 \mathrm{~m} \times 3 \mathrm{~m}$, step-size of 5 degrees, and the two 
TABle 2: Average position error (in meters) for the layout of Figure 3(b) (array size $=8$, step-size $=5$ degrees).

\begin{tabular}{|c|c|c|c|c|c|c|c|c|c|}
\hline & \multicolumn{3}{|c|}{ Radio map res $1 \times 1$} & \multicolumn{3}{|c|}{ Radio map res $2 \times 2$} & \multicolumn{3}{|c|}{ Radio map res $3 \times 3$} \\
\hline & $\mathrm{AP}=1$ & $\mathrm{AP}=2$ & $\mathrm{AP}=3$ & $\mathrm{AP}=1$ & $\mathrm{AP}=2$ & $\mathrm{AP}=3$ & $\mathrm{AP}=1$ & $\mathrm{AP}=2$ & $\mathrm{AP}=3$ \\
\hline SPS-L1 & 0.73 & 0.54 & 0.53 & 2.45 & 1.52 & 1.40 & 3.45 & 1.78 & 1.64 \\
\hline SPS-L2 & 0.81 & 0.54 & 0.53 & 2.52 & 1.49 & 1.39 & 3.54 & 1.79 & 1.66 \\
\hline SPS-EMD & 0.89 & 0.53 & 0.51 & 2.56 & 1.54 & 1.34 & 3.54 & 2.00 & 1.69 \\
\hline SPS-PPD & 1.13 & 0.59 & 0.84 & 2.71 & 1.63 & 1.65 & 3.86 & 1.89 & 1.77 \\
\hline SPS-HD & 1.28 & 0.67 & 0.61 & 2.91 & 1.77 & 1.27 & 4.08 & 2.00 & 1.80 \\
\hline SPS-KL & 1.21 & 0.75 & 0.57 & 2.93 & 1.70 & 1.49 & 3.88 & 2.45 & 2.20 \\
\hline RSS-L2 & 5.87 & 3.87 & 1.86 & 5.95 & 3.95 & 2.37 & 5.98 & 3.97 & 3.00 \\
\hline
\end{tabular}

TABLE 3: Average position error for a single room $16 \times 16$ (array size $=8$, step-size $=5$ degrees).

\begin{tabular}{lccr}
\hline & $\begin{array}{c}\text { Radio map } \\
\text { resolution } 1 \mathrm{~m} \times 1 \mathrm{~m}\end{array}$ & $\begin{array}{c}\text { Radio map } \\
\text { resolution } 2 \mathrm{~m} \times 2 \mathrm{~m}\end{array}$ & $\begin{array}{c}\text { Radio map } \\
\text { resolution } 3 \mathrm{~m} \times 3 \mathrm{~m}\end{array}$ \\
\hline SPS-L1 & 0.47 & 0.91 & 1.48 \\
SPS-L2 & 0.52 & 0.95 & 1.52 \\
SPS-EMD & 0.43 & 0.83 & 1.3 \\
SPS-PPD & 0.44 & 0.79 & 1.21 \\
SPS - HD & 0.67 & 0.96 & 1.49 \\
SPS - KL & 1.34 & 1.78 & 2.30 \\
Lower bound $(\mathbf{m})$ & $\mathbf{0 . 3 9}$ & $\mathbf{0 . 7 6}$ & $\mathbf{1 . 1 8}$ \\
\hline
\end{tabular}

metrics L1 and PPD, this relationship has been displayed in Figure 12. It should be noted that for a given radio frequency, the radius of the circular array is proportional to the number of array elements. Therefore, large array sizes might create practical implementation issues.

Another issue with the SPS-based approach is the complexity in terms of the amount of storage required for the radio-map. The number of samples in one spatial spectrum depends on the number of azimuth angles that the received power has been measured for. This is directly controlled by the step-size chosen to electronically rotate the main beam of the receiver antenna. Smaller step-size amounts to large number of samples per SPS. Consequently, large amount of storage is required for the radio-map. In addition, the operational speed of the SPS matching process will decrease when the step-size is small. As it is desirable to maximize the speed and at the same time minimize the storage requirement, it is interesting to see the effect of large step-sizes on the accuracy of the SPS-based system. Note that for a given number of antenna elements, the step-size basically describes the resolution of an SPS. Figure 13 displays the variation in the average position error as the rotation step-size increases (i.e., SPS resolution decreases). Compared to a 5-degree step-size, resolution of the spatial spectra obtained by a 40 degree rotation step-size reduces by a factor of 8 ; however, as seen in Figure 13 , only a modest rise in average error is observed. It is interesting to note that an 8-element circular array antenna with beamforming capability and a rotation step size of 45 degrees is almost equivalent to a sectorized antenna with 8 sectors. Sectorized antennas are basically a special case of the general methodology outlined so far.
Another interesting point in Figure 13 is that larger array sizes (e.g., 12 or 16) exhibit lower average errors when the SPS resolution is high, yet smaller array sizes (e.g., 4 or 8) perform better at higher step-sizes.

In all the results provided so far, we have used the beam pattern generated by a simple circular phased-array antenna with no side-lobe suppression. It is worth noting that the beam patterns generated in this way are not quite identical when the main lobe is pointing at different directions. This fact has been incorporated in our simulations to generate proper spatial spectrum per transmitter location. We have also performed simulations with ideal beam pattern with no side-lobes. There was no considerable change in the overall system performance and for this reason those results have been omitted. In fact, using ideal beam pattern only changes the nature of the observed spatial spectra. The SPS observed at the receiver as well as all recorded signatures in the radiomap will be different. However, ultimately, the performance of the system depends on how efficiently the closest signatures are selected from the radio map. Since, this process is not changed; similar performance is obtained even if ideal beam pattern is considered.

\section{CONCLUSION}

The underlying philosophy in this paper is that exploiting the information in the spatial distribution of RF energy around a receiver results in better estimates of the location of a mobile. This spatial spectrum basically represents a signature that only depends on the relative location of the transmitter with respect to the receiver and the environment surrounding 
TABLe 4: Average position error for a single room $32 \times 32$ (array size $=8$, step-size $=5$ degrees)

\begin{tabular}{lccc}
\hline & $\begin{array}{c}\text { Radio map } \\
\text { resolution } 2 \mathrm{~m} \times 2 \mathrm{~m}\end{array}$ & $\begin{array}{c}\text { Radio map } \\
\text { resolution } 4 \mathrm{~m} \times 4 \mathrm{~m}\end{array}$ & $\begin{array}{c}\text { Radio map } \\
\text { resolution } 6 \mathrm{~m} \times 6 \mathrm{~m}\end{array}$ \\
\hline SPS-L1 & 0.99 & 1.81 & 2.79 \\
SPS-L2 & 1.10 & 1.86 & 2.93 \\
SPS-EMD & 0.92 & 1.65 & 2.45 \\
SPS-PPD & 0.84 & 1.61 & 2.38 \\
SPS-HD & 1.21 & 1.94 & 2.83 \\
SPS-KL & 2.04 & 4.10 & 4.31 \\
Lower bound $(\mathbf{m})$ & $\mathbf{0 . 7 8}$ & $\mathbf{1 . 5 3}$ & $\mathbf{2 . 2 7}$ \\
\hline
\end{tabular}

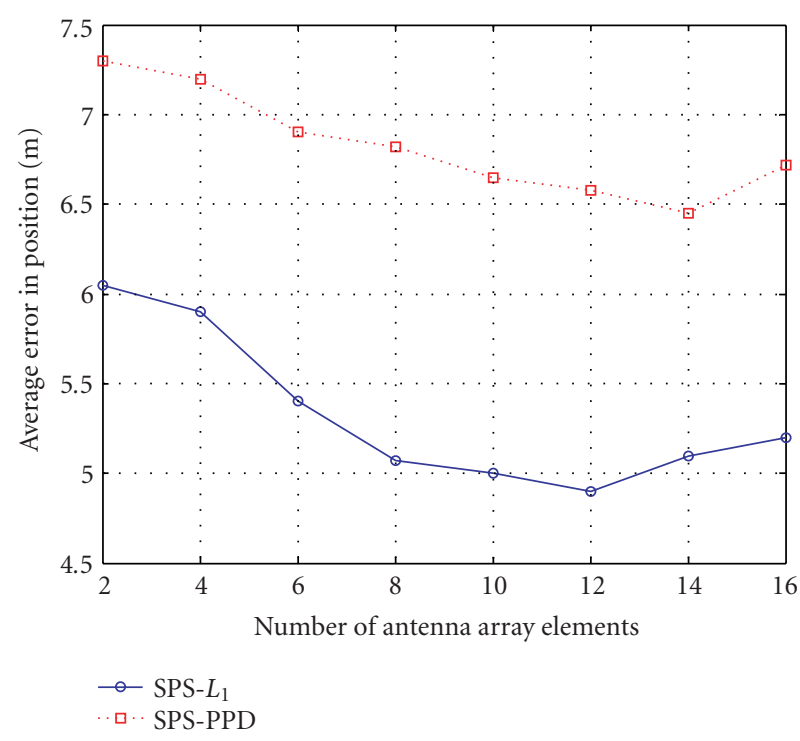

FIGURE 12: Average error versus antenna array size (1000 test mobile positions).

them. It can be easily seen that in free space, there is a oneto-one correspondence between the transmitter position and the received SPS. If the receiver is assumed to be at the origin of a polar coordinate system, the received spatial signature is a function of the polar coordinate of the transmitter. If the receiver-transmitter pair is planted inside a building, the layout and the construction material of the walls dictate the flow of energy; and therefore, the shape of the signature. However, the uniqueness of the SPS signatures is still maintained in an indoor environment. Therefore, if a database consisting of a set of representative points (i.e., radio-map) in a building is made, then, any inquiry to the whereabouts of a mobile can be answered by comparing the received SPS with the entries of the radio-map.

RSS-based methodologies also follow the same strategy; however, for them to have a reasonable accuracy radio visibility of the mobile by at least three access points is required. This would create a difficult coverage design problem, which would be eliminated if SPS signatures were used instead. In

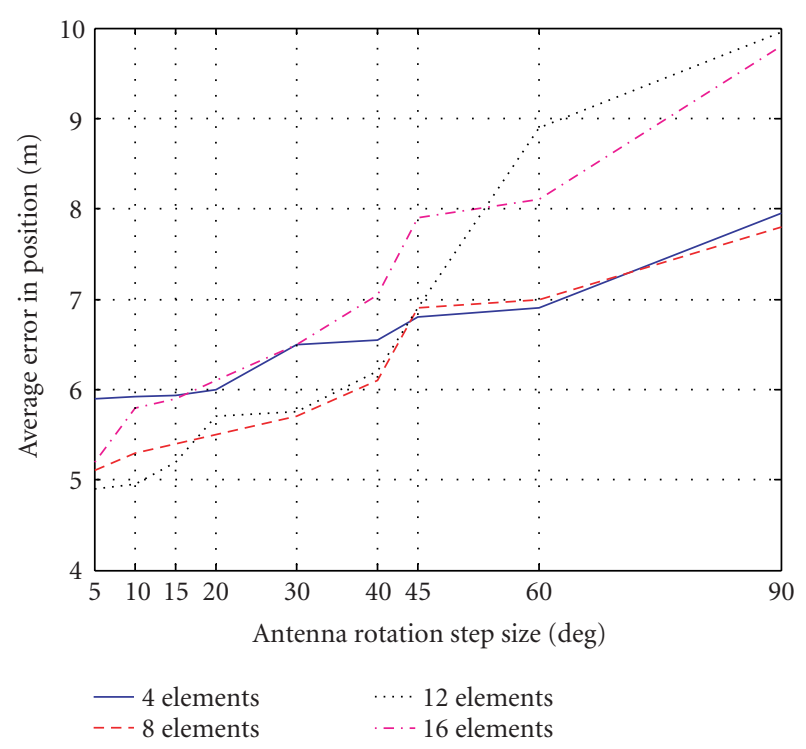

FIGURE 13: Average position error for various step-sizes (building in Figure 3(a), radio-map $3 \times 3 \mathrm{~m}$ ).

other words, an advantage of using the SPS signatures as opposed to RSS (i.e., pure signal strength) is that even single receiver with beamforming capability delivers good accuracy; and as a result, complicated triple coverage by three access points is no longer required. Theoretically speaking, if the capability of estimating both direction and range of a mobile exists, then, only one access point is enough to estimate the position of any mobile transmitter. However, to the best of our knowledge, no known methodology currently exists that is capable of providing reasonable and simultaneous estimate of direction and range information in indoor environments. Specifically, our previous research has shown that the direction of a mobile can only be estimated with $40 \%$ to $70 \%$ probability (depending on the material of the walls) within 20 degrees of error inside buildings. Therefore, we do not wish to rely on estimating angle-of-arrival (AOA) in our positioning system unlike the methodology outlined in [19].

It is important to note that in practice the effective radiation pattern of the transmitter antenna is not 


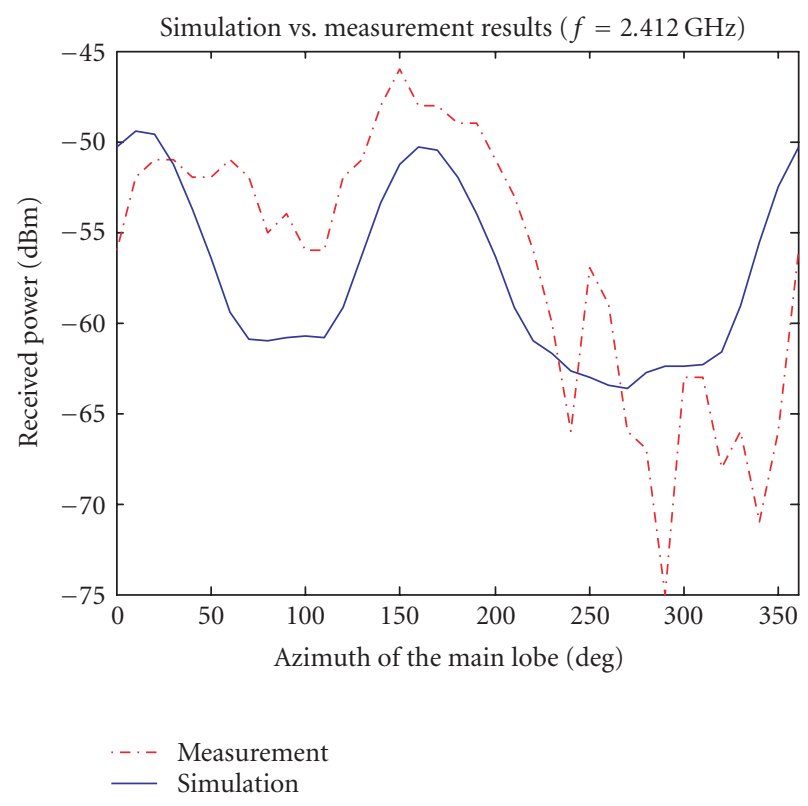

FIGURE 14: Comparison of the measured SPS with the ray-tracing estimate.

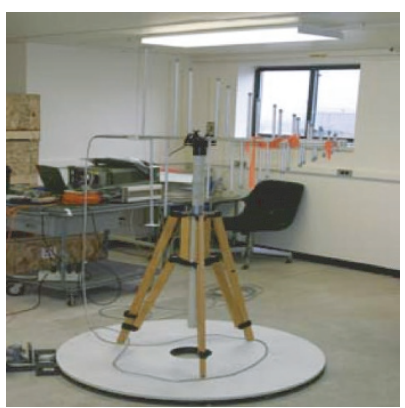

(a)

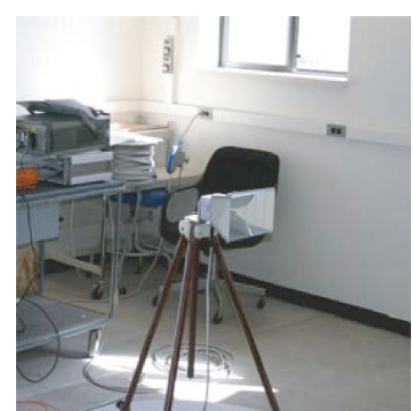

(b)

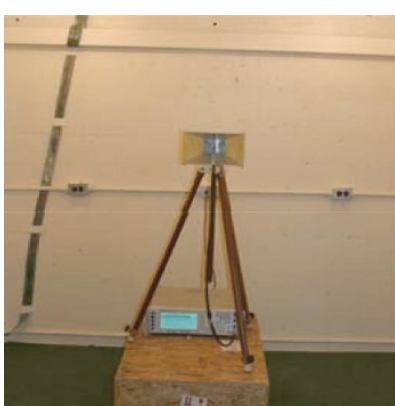

(c)

FIGURE 15: Hardware experiment to validate the SPS generated by the ray-tracing tool.

omnidirectional. This is mostly due to hardware issues and body absorption if a person is carrying the transmitter. This will have an impact on the performance of any positioning system that relies on the signal strength. The extent of this impact is difficult to address in simulation. As, there are also many other factors such as movement of other people or objects in the building that could also affect the results, hardware implementation is the best way to characterize and evaluate this impact. When building a radio-map, a simple technique to reduce the body effect is to measure four spatial spectra while the person that carries the transmitter faces 4 different directions such as north, south, west, and east. All four spatial spectra or their average can be used per transmitter location. Again, actual hardware measurements are recommended to exactly assess the performance of the system.

Throughout this paper, we have considered the case where the stationary access point with the circular array is the receiver and the mobile is the transmitter. If there are many transmitters available, a multiple access scheme has to be in place to differentiate between the signals of different transmitters. It is also possible to consider the case where the mobile is the receiver capable of generating spatial signatures; however, it should be noted that in this case, the mobile requires an electronic compass (or a similar device) in order to have a reference frame for the azimuth angle. In this way, many mobiles can estimate their locations simultaneously without the need for a multiple access scheme.

The application of multiple-in-multiple-out (MIMO) technology in WLAN (e.g., 802.11n) is creating the possibility to have access points that are capable of creating beam patterns that adapts to user's location. Access points that can select the best directional beam pattern among a finite number of possible patterns are already making their way into the market. It is conceivable that a simplified version of our proposed approach can be build over such infrastructure. 
Finally, although we have outlined several matching techniques in this paper, it should be noted that our primary objective is to show the feasibility and effectiveness of using SPS for indoor positioning. Further studies are required to find the optimal strategy by considering other signature matching methodologies, operational complexity, and implementation issues.

\section{APPENDIX \\ COMPARISON OF THE SIMULATION SPS WITH REAL MEASUREMENTS}

In order to justify the use of the ray-tracing tool for performance analyses of the SPS-based approach, verification of the accuracy of the simulated spatial power spectrum with real measurements was necessary. Therefore, a simple experiment was set up that involved a directional antenna located on a rotating platform. The SPS was generated by taking the measurement of the received power when the azimuth angle of the directional antenna was pointing at $0,10,20, \ldots, 350$ degrees. Figure 14 demonstrates the comparison made between the measured SPS and the ray-tracing estimate. The measured SPS corresponds to a single physical coordinate; and therefore, exhibits the effects of multipath fading. By averaging the sample SPS corresponding to a few nearly colocated positions, a closer match to the smoothed outcome of the ray-tracing tool was observed. Averaging four such measurement samples provided good match with the ray-tracing results. Details of the hardware experiment (Figure 15) have been omitted for brevity. The authors realize that in general such hardware measurements should replace the results obtained by the ray-tracing tool to further validate our conclusions.

\section{ACKNOWLEDGMENTS}

The authors would like to express their gratitude to Dr. Kate Remely, Mike Mckinley, and Marc Rutschlin from the Electromagnetic Division of the National Institute of Standard and Technology located in Boulder Colorado for providing the measurement results of the rotating directional antenna. Also, the authors would like to thank the anonymous reviewers for their valuable comments.

\section{REFERENCES}

[1] J. Hightower and G. Borriello, "Location systems for ubiquitous computing," IEEE Computer Magazine, vol. 34, no. 8, pp. 57-66, 2001.

[2] P. Bahl and V. N. Padmanabhan, "RADAR: an in-building RFbased user location and tracking system," in Proceedings of the 19th Annual Joint Conference of the IEEE Computer and Communications Societies (INFOCOM '00), vol. 2, pp. 775-784, Tel Aviv, Israel, March 2000.

[3] A. M. Ladd, K. E. Bekris, G. Marceau, A. Rudys, D. S. Wallach, and L. E. Kavraki, "Using wireless ethernet for localization," in Proceedings of IEEE/RSJ International Conference on Intelligent Robots and System (IROS '02), vol. 1, pp. 402-408, SeptemberOctober 2002.
[4] A. M. Ladd, K. E. Bekris, A. P. Rudys, D. S. Wallach, and L. E. Kavraki, " On the feasibility of using wireless ethernet for indoor localization," IEEE Transactions on Robotics and Automation, vol. 20, no. 3, pp. 555-559, 2004.

[5] E. Elnahrawy, X. Li, and R. P. Martin, "The limits of localization using signal strength: a comparative study," in Proceedings of 1st Annual IEEE Communications Society Conference on Sensor and Ad Hoc Communications and Networks (SECON '04), pp. 406-414, Santa Clara, Calif, USA, October 2004.

[6] H. Hashemi, "The indoor radio propagation channel," Proceedings of the IEEE, vol. 81, no. 7, pp. 943-968, 1993.

[7] Q. H. Spencer, B. D. Jeffs, M. A. Jensen, and A. L. Swindlehurst, "Modeling the statistical time and angle of arrival characteristics of an indoor multipath channel," IEEE Journal on Selected Areas in Communications, vol. 18, no. 3, pp. 347-360, 2000.

[8] R. B. Ertel, P. Cardieri, K. W. Sowerby, T. S. Rappaport, and J. H. Reed, "Overview of spatial channel models for antenna array communication systems," IEEE Personal Communications, vol. 5, no. 1, pp. 10-22, 1998.

[9] S. J. Fortune, D. M. Gay, B. W. Kernighan, O. Landron, R. A. Valenzuela, and M. H. Wright, "WISE design of indoor wireless systems: practical computation and optimization," IEEE Computational Science and Engineering, vol. 2, no. 1, pp. 5868, 1995.

[10] R. A. Valenzuela, O. Landron, and D. L. Jacobs, "Estimating local mean signal strength of indoor multipath propagation," IEEE Transactions on Vehicular Technology, vol. 46, no. 1, pp. 203-212, 1997.

[11] Y. Rubner, C. Tomasi, and L. J. Guibas, "A metric for distributions with applications to image databases," in The 6th International Conference on Computer Vision, pp. 59-66, Bombay, India, January 1998.

[12] G. A. Edgar, Measure, Topology, and Fractal Geometry, Springer UTM, New York, NY, USA, 1995.

[13] P. K. Agarwal and M. Sharir, "Efficient algorithms for geometric optimization," ACM Computing Surveys, vol. 30, no. 4, pp. 412-458, 1998.

[14] D. P. Huttenlocher and W. J. Rucklidge, "A multi-resolution technique for comparing images using the Hausdorff distance," in Proceedings of IEEE Computer Society Conference on Computer Vision and Pattern Recognition (CVPR '93), pp. 705706, New York, NY, USA, June 1993.

[15] W. J. Rucklidge, "Efficiently locating objects using the Hausdorff distance," International Journal of Computer Vision, vol. 24, no. 3, pp. 251-270, 1997.

[16] S. Kullback and R. A. Leibler, "On information and sufficiency," Annals of Mathematical Statistics, vol. 22, pp. 79-86, 1951.

[17] Y. Stylianou and A. K. Syrdal, "Perceptual and objective detection of discontinuities in concatenative speech synthesis," in Proceedings of IEEE International Conference on Acoustics, Speech, and Signal Processing (ICASSP '01), Salt Lake City, Utah, USA, May 2001.

[18] J. Goldberger, S. Gordon, and H. Greenspan, "An efficient image similarity measure based on approximations of KLdivergence between two Gaussian mixtures," in Proceedings of 9th IEEE International Conference on Computer Vision, vol. 1, pp. 487-493, Nice, France, October 2003.

[19] D. Niculescu and B. Nath, "VOR base stations for indoor 802.11 positioning," in Proceedings of the 10th Annual International Conference on Mobile computing and networking (MobiCom '04), pp. 58-69, Philadelphia, Pa, USA, SeptemberOctober 2004. 
Kamran Sayrafian-Pour (Senior Member, IEEE) received his B.S., M.S., and Ph.D. degrees in electrical and Computer engineering from Sharif University of Technology, Villanova University, and the University of Maryland in 1989, 1993, and 1999, respectively. From 1996 to 1998, he was employed at LCC Int., McLean, Virginia, where he worked on the design and simulation of cellular and personal communication net-

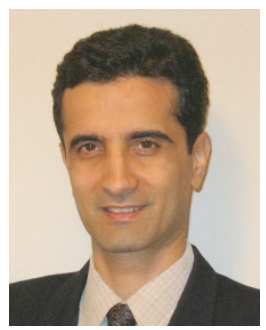
works; he also served as a Faculty Member for LCC Corporate University where he was involved in teaching and developing various courses related to wireless communication systems. In 2000, he cofounded Zagros Networks, Inc., a fables semiconductor company based in Rockville, Maryland, where he served as President and Senior Member of the architecture team. Dr. Sayrafian-Pour is an Adjunct Faculty of the University of Maryland, College Park, since 2003. He has also been a Member of the Wireless Communications Technologies Group at the National Institute of Standards and Technology, Gaithersburg, Maryland, since 2004. His current research areas include application of smart antennas in wireless communication systems, multiple access techniques, and mobile ad hoc networks.

Dominik Kaspar received his M.S. degree in computer science from the Swiss Federal Institute of Technology (ETH), Zürich, in 2005. From 2003 to 2005, he worked for the National Institute of Standards and Technology, Maryland, USA, as a Guest Researcher. His research interests include indoor positioning systems, modeling, and simulation of wireless networks.

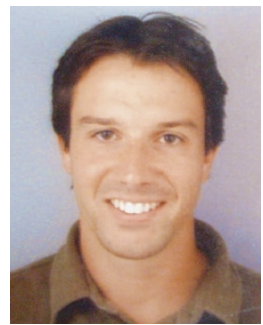

